\title{
SOLAR RADIO BRIGHT SPOTS AT 88-CM WAVELENGTH
}

\author{
J. FIROR \\ Department of Terrestrial Magnetism, Carnegie Institution \\ Washington, D.C., U.S.A.
}

\section{INTRODUCTION}

During 1957 we studied the solar radio radiation at a wavelength intermediate between the centimeter region with its slowly varying bright spots and the meter-wavelength region with its noise storms. At this intermediate wavelength $(88 \mathrm{~cm})$ the slowly varying bright-spot and the noise-storm producing regions merge into two aspects of the same persistent, bright, solar radio regions.

\section{PERSISTENT BRIGHT SPOTS}

The source of the slowly varying bright regions on the sun was described by Covington [1] in 1954 in terms of Waldmeier's coronal condensations and of his own work, with Miss Dodson, that showed the close relation of $10-\mathrm{cm}$ radiation and bright plages. To quote Covington: "One might say that the radio sunspot is such a condensation with the bright plage and dark sunspot at the base."

Since 1954 no new evidence has appeared to change this picture very much, while the close relation of the centimeter and decimeter radiation with calcium plages has been confirmed by others [2].

At meter wavelengths there are also bright regions that persist for several days [3]. These regions differ from the slowly varying regions in their having rapid intensity changes and in their not being closely associated with optical active regions. Boischot has shown, from the $177-\mathrm{cm}$ observations, that although one can sometimes relate an active radio region with an active optical region, there is no permanent geometrical relation between the two [4].

Our observations have been made in the intermediate $88-\mathrm{cm}$ wavelength region. The antenna used was a periodic array of helical elements giving fan beams 4.8-minutes-of-arc wide to half-power points. Fig. 1 shows scans with several bright regions-some quiet, some the source of bursts-passing across the disk.

The quiet bright spots in Fig. 1 strongly resemble the quiet spots of the slowly varying component. These regions persist for several days or more as do the slowly varying spots, and the day-to-day changes in intensity are similar to those seen at $21 \mathrm{~cm}$ [2]. The positions on the solar disk of these regions in general agree with the positions of the large active regions when 
both are near the central meridian. Furthermore, the apparent temperature of the regions whose diameter can be measured is in the range $10^{6}$ to $10^{7}{ }^{\circ} \mathrm{K}$, which agrees with the results at $60 \mathrm{~cm} \mathrm{[5]} \mathrm{and} \mathrm{at} \mathrm{shorter} \mathrm{wavelengths.} \mathrm{Thus}$ it seems likely that the quiet bright spots seen at $88 \mathrm{~cm}$ are associated with the regions of the slowly varying component. This result implies that the source of these regions at least some of the time reaches sufficiently high in the solar atmosphere to be seen at $88 \mathrm{~cm}$.

Some regions are not quiet but produce numerous bursts. Fig. 1 shows some of these regions, and Fig. 2 shows three larger examples. Although the position line for the active radio spot on the disk agrees with an optical region for the first and last case in Fig. 2, there is no optical region along the position line for the strongest storm of the three. That these regions are actually the noise storms of the meter wavelengths is shown by comparisons with sweep-frequency records. When the spot seen on the $88-\mathrm{cm}$ scan is sufficiently intense, the characteristic type I bursts are seen on the sweep-frequency records [6]. But, on the $88-\mathrm{cm}$ record, there is no clear distinction between these stormy regions and the slowly varying ones. A quiet spot can become noisy; a noisy spot can become quiet. Although the active radio spots may be more intense than the quiet ones, the smallest recognizable active spot is just an occasional burst occurring in an otherwise quiet bright region. In every case in which a noise storm has been observed to begin on the disk, the brightening has occurred in a preexisting quiet bright spot.

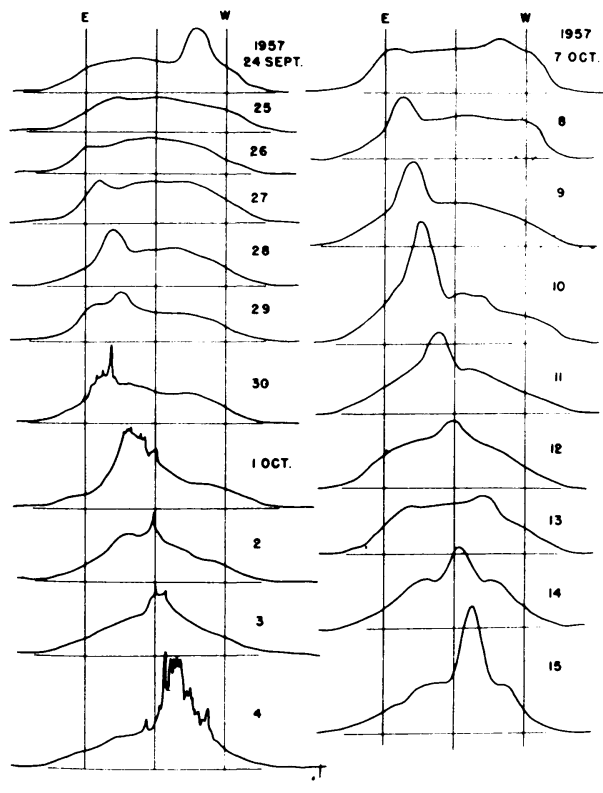

FIG. 1. Successive daily strip scans of the sun at $88-\mathrm{cm}$ wavelength for two periods in 1957 .

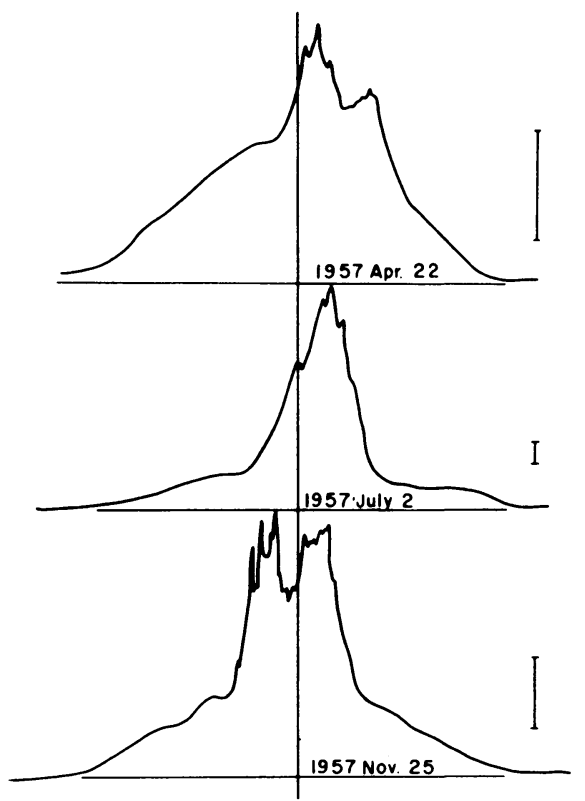

Fig. 2. Three examples of noise storms as seen by 88 -cm strip scans. 
Thus, at $88-\mathrm{cm}$ wavelength, the noise storms of the meter wavelengths and the slowly varying bright spots at the decimeter wavelength become just two different aspects of the persistent bright spots.

A model that allows these results to be simply displayed can be constructed from the coronal condensation having a plage for a base. This condensation, which can occasionally extend high enough to be seen at $88 \mathrm{~cm}$, is now imagined to be, itself, the base of a more extensive structure. This extension of the condensation is then the seat of noise-storm activity. The sporadic occurrence of noise storms requires some additional agency to be operating. The work of Payne-Scott and Little [7] some years ago points to the existence of a large optical spot, and hence of large magnetic fields, as this additional agency. Fig. 3 shows, schematically, the resulting picture of a complete active region. A model of this sort is consistent with the sequence of events seen when a single active region developed on an otherwise quiet sun in 1954 [8]. The great and variable heights in the solar atmosphere observed for meter-wavelength noise storms (up to one solar radius above the photosphere) suggest that this model need not require a close positional association between the source of the meter-wavelength noise storm and the optical active region or the decimeter-wavelength radio bright spot.

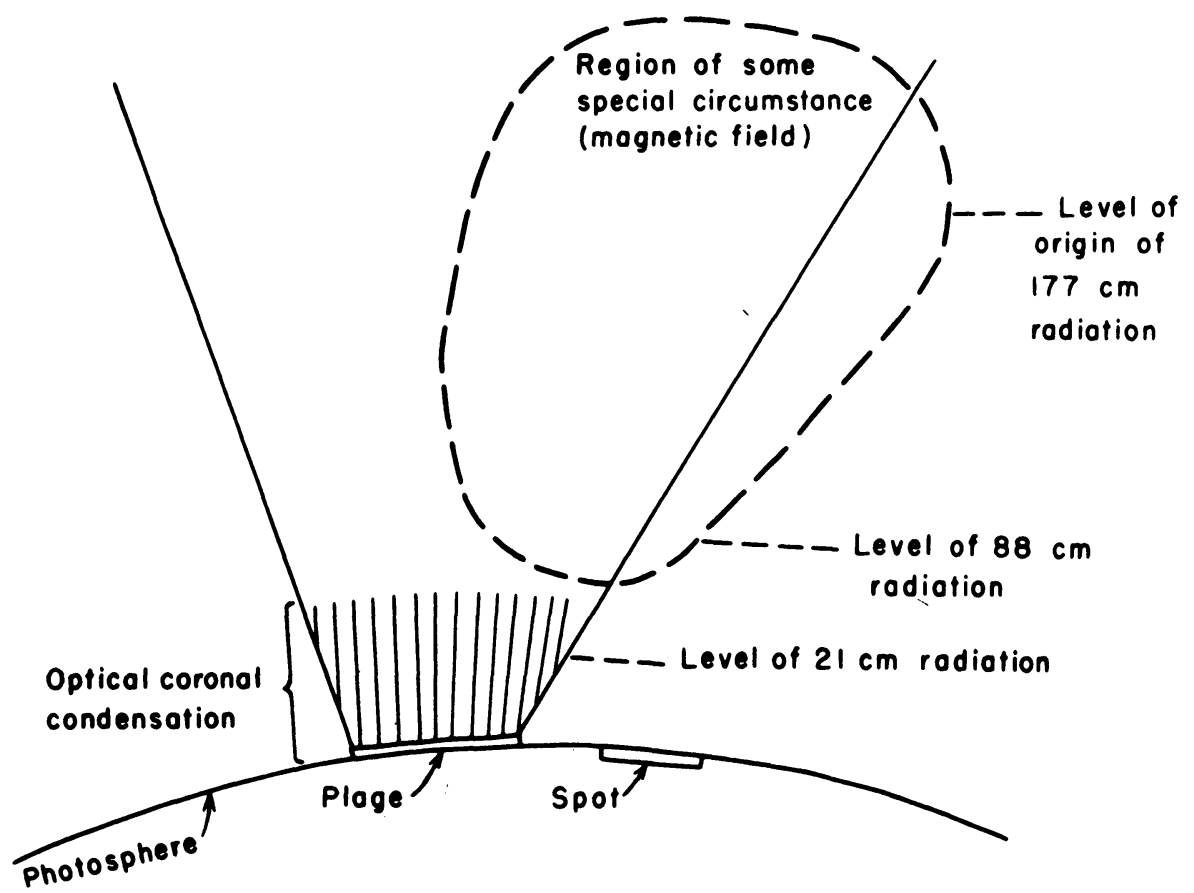

FIG. 3. Schematic diagram of an active solar region that produces both slowly varying bright spots at centimeter wavelengths and noise storms at meter wavelengths. 


\section{REFERENCES}

[1] Covington, A. E. J. Geophys. Res. 59, 166, 1954.

[2] Christiansen, W. N., Warburton, J. A., and Davies, R. D. Aust. J. Phys. 10, 491, 1957.

[3] Avignon, Y., et al. C. R. 244, 1460, 1957.

[4] Boischot, A. Thesis, Univ. of Paris, 1958.

[5] Swarup, G., and Parthasarathy, R. Program of URSI Meeting, Washington, D.C., April 1958.

[6] I am indebted to Professor Fred Haddock of the University of Michigan for the use of his sweep-frequency records.

[7] Payne-Scott, R., and Little, A. G. Aust. J. Sci. Res. A 4, 508, 1951.

[8] Dodson, H. W., and Hedeman, E. R. M.N.R.A.S. 116, 428, 1956. 